\title{
The disk targets for LAMOST pilot survey
}

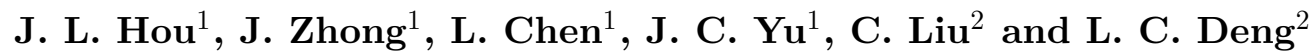 \\ ${ }^{1}$ Shanghai Astronomical Observatory, CAS, Shanghai, China \\ email: houjl, jzhong, chenli, yujc@shao.ac.cn \\ ${ }^{2}$ National Astronomical Observatory, CAS, Beijing, China \\ email: chaoliu@lamost.org, licai@nao.ac.cn
}

\begin{abstract}
LAMOST has 4 meters in diameter with 4000 fibers in the focal plane. The telescope is now on the way towards full spectroscopy survey periods. This survey project has the potential to largely increase our understanding of the substructure of the Galactic stellar spheroid and different disk components through accurate measurements of radial velocities, metallicities and effective temperatures of millions of stars.
\end{abstract}

Keywords. Galaxy: disk — techniques: spectroscopic — surveys

A low latitude disk survey strategy for the disk pilot survey was proposed. We focused on the regions that have open clusters which are mainly aimed at testing the survey efficiency and data quality. We use the PPMXL astrometric catalog, which provides positions, proper motions, B/R/I magnitudes (mostly) from USNO-B and $\mathrm{J} / \mathrm{H} / \mathrm{Ks}$ from 2MASS as well. In order to constrain our targets below the dec $=60 \mathrm{deg}$, we have chosen 8 plates along the galactic plane. RA from about 0 to $67 \mathrm{deg}$, DEC varies for each plate, from 42 to $59 \mathrm{deg}$. Adjacent plates may have small overlaps. Each plate covers an area of $2.5 \mathrm{deg}$ radius, with a central star (for Shark-Hartmann guider) brighter than $\sim 8$ th magnitude. For each plate, the created input catalog has stars with $11.3<\operatorname{Imag}<16.3$ and Bmag available from PPMXl. The stars are selected according to special selection functions that satisfy the requirements of the fiber positioning and uniform distribution in color-color diagram, details can be found in Chen et al. (2012).

Our final input catalog consists of 8 plates, each about 12000 stellar objects. In Figure 1 , we show the positions of 8 plates that were chosen for the pilot survey.

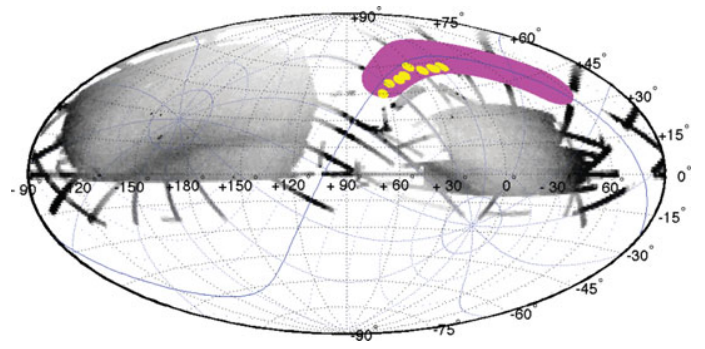

Figure 1. Eight circles are the pilot survey regions for the Galactic disk.

This work was supported by the NSFC grants 11173044 (PI Hou), 11073038 (PI Chen), Key Project 10833005 (PI Hou)

\section{Reference}

Chen L., Hou J. L., Yu J. C., Liu C., \& Deng L. C. etc. 2012, RAA, 12(7), 805 